\title{
Docile Avatars: Aesthetics, Experience, and Sexual Interaction in Second Life
}

\author{
Shaowen Bardzell \\ 535 W. Michigan Street \\ School of Informatics \\ Indiana University \\ Indianapolis, Indiana 46202 \\ selu@indiana.edu
}

\author{
Jeffrey Bardzell \\ $1900 \mathrm{E} 10^{\text {th }}$ Street \#938 \\ School of Informatics \\ Indiana University \\ Bloomington, IN 47406 \\ jbardzel@indiana.edu
}

\begin{abstract}
Second Life, a participant-created multi-user virtual environment (MUVE), gained sudden media acclaim in 2006. Prior to that, the world was developing many of the characteristics that have come into their own today, such as virtual fashion lines, a thriving virtual economy, scripted interactive furniture, vehicles, and toys. Perhaps not surprisingly, much of the early content was adult in nature, from cyberstrip clubs to kinky lingerie, sex animations, and interactive virtual genitalia. More surprising was the visibility and prevalence of the BDSM (bondage, discipline, and sadomasochism) subculture. In this paper, we report results from a two-year study of the BDSM subculture in Second Life, combining virtual ethnography and artifact analysis with recent HCI theories of experience design to understand how and why this complex phenomenon emerged from Second Life users. We contend that the participant-created world enables the construction of powerful aesthetic experiences, and that these experiences are made possible by the interweaving of visual, literary, and interaction aesthetics.
\end{abstract}

\section{Categories and Subject Descriptors}

H.5.1 [Information Interfaces and Presentation (e.g., HCI)]: Multimedia Information Systems - artificial, augmented, and virtual realities.

\section{General Terms}

Design, Human Factors.

\section{Keywords}

Aesthetics, experience, interface, interaction, BDSM.

\section{INTRODUCTION}

Anyone paying the slightest attention to virtual worlds knows that sex is a major part of them, in particular, more fetishized forms of sex, including cyberstripping and escorts, furry sex (sex between humanoid-animal avatars), and BDSM (or bondage, dominance and submission, and sadomasochism). Virtual world sex is ubiquitous, appearing in fantasy-themed commercially created worlds such as World of Warcraft [27].

(C) Shaowen Bardzell, Jeffrey Bardzell, 2007

Published by the British Computer Society

People and Computers XXI-HCI... but not as we know it:

Proceedings of HCI 2007

Linden J. Ball, M. Angela Sasse, Corina Sas, Thomas C. Ormerod, Alan Dix, Peter Bagnall, and Tom McEwan (Editors)
But sex, especially fetish sex, comes into its own in multi-user virtual environments (MUVEs) with user-created content, such as Second Life, There, and ActiveWorlds. There are obvious reasons for the popularity of fetish sex in these worlds: they can be considerably less expensive than real-life fetish sex (rubber outfits, whips, restraints, and swings add up); the anonymity of these worlds frees people to act without the usual social restraints; and the increasingly compelling visuals of these environments provide high quality visual stimulation.

Yet the obvious explanations fail to account for the broad appeal of a subculture, such as that of BDSM, in the first place. We believe that metaverses such as Second Life provide new interfaces to a classic, if taboo, aesthetic. BDSM is often understood as a form of sexual practice, especially in academic literature $[24,39]$. The practitioner literature $[1,40]$ commonly extends the practice to a 'lifestyle', which in addition to sex also includes regular household and even public/social interactions; this literature also typically derides the commonly held notion that dominance and submission $(\mathrm{D} / \mathrm{s})$ is little more than a form of kinky sex.

What is the experience of online sexual interaction, especially virtual BDSM, beyond a type of sexual practice? Through ethnography and analysis of virtual artifacts, we have seen that participants view virtual BDSM not as a sexual practice, but rather as a full-blown aesthetic, and that its sexual practices are a part of that aesthetic. By 'aesthetic', as we elaborate below, we mean a particular kind of experience that arises when, in the midst of a complex human struggle, one finds meaning, which creates a sensation of self-transcendence and the desire to articulate and share it with others via cultural artifacts.

We contend that virtual worlds enable the construction of a unique fetish sex experience, which is connected to, yet distinct from, the real-life BDSM aesthetic. More importantly, the differences can largely be explained by structural forces associated with computer interfaces themselves. As noted earlier, members of a community share a set of values; these values are embodied in rituals, social interactions, and artifacts. In virtual worlds, all three of these embodiments are computermediated. Speech is mediated through an IM window; leather and lace are equally made of pixels; furniture and sex toys are mediated through clickable objects; these objects are accessed from a Windows Explorer/directory tree 'Inventory' interface; body parts, such as hair, genitalia, and nipples can only be touched through dialog boxes; enterprising partners who want to design their own sexual experiences often have to write scripts in the C-like Linden Scripting Language (LSL), though some vendors facilitate this process by selling generic, customizable scripts. Whatever artistry there may be to the construction of a BDSM sexual encounter, its theatre, its 
thematic coherence, its embodied imagination, all of it is mediated by computer interfaces. Interface and aesthetics are mutually interconnected.

To explore the mutual relationships between the interface and aesthetics of BDSM, we consider the interfaces using the traditional aesthetic categories of visual aesthetic, literary aesthetic, and the interaction aesthetic. These are examined using notions of 'aesthetic' and 'experience' derived from recent HCI literature on experience design, which in turn refer back to the pragmatism of Dewey, the hermeneutics of Dilthey, and the anthropology of Turner.

\section{TOWARD AN AESTHETICS OF INTERACTION}

In the past decade, the field of HCI has become increasingly preoccupied with the personal, the embodied, and the subjective. This preoccupation has come in different forms and under different names, including affective computing [29], experience design [6,9], emotional design [26], intimate computing [4], embodied interaction [13], and even sexual interactions [5, 7]. In short, $\mathrm{HCI}$ is increasingly concerned with embodied and phenomenological, rather than rationalist, accounts of human (inter)action. One of these accounts is to emphasize the aesthetics of experience.

\subsection{Theorizing 'Aesthetic Experience'}

Aesthetics is a hotly contested tradition that spans more than two millennia in the West. In general terms, it deals with two related theorizations, that of beauty and that of art, and has wrestled with questions such as whether beauty is a property of certain aesthetic objects, as assumed by eighteenth-century British theorists, or whether it is a special subjective response that can be had to any object, so long as it is experienced in a certain way, a position advocated by Schopenhauer [12]. Though later thinkers vary in their explanation of the exact mechanisms of how it all works, the notion that the aesthetic is connected to experience, rather than artifacts themselves, has persisted, to the point where, as in the case of Dewey, 'experience' and 'aesthetic' become difficult to distinguish.

Late nineteenth- and early twentieth-century German philosopher Wilhelm Dilthey, credited with a revival in hermeneutics that would go on to influence phenomenology, stressed 'lived experience', in contrast to Cartesian disembodied rationalism, as the primary reality. Lived experience, for Dilthey, includes not only perception and cognition, but also subjective phenomena, such as emotions, fears, and desires [8]. In elaborating this theory, Dilthey, as summarized in $[8,35]$ distinguishes between external reality, individual experience, and cultural expressions (such as novels and plays). The expressions-a given culture's meaningful artifacts-link individual experiences and create the culturally distributed framings of experiences that underlies cultural cohesion. This approach advocates the use of cultural artifacts to understand relations between external reality and people's subjective experience of them.

American pragmatist John Dewey's Art as Experience contains a sustained analysis of experience, which builds on a distinction of Dilthey between 'experience' and 'an experience'. Experience is simply the succession of things that happen to us; in contrast, an experience is a single meaningful experience, having a beginning and an ending, a coherent unity or "single quality'; experience can only be complete and whole if it is interpreted (i.e., transformed into an experience), and as an interpreted, meaningful whole, it is capable of being articulated into a cultural expression [11]. It is accompanied by "a satisfying emotional quality because it possesses internal integration and fulfillment reached through ordered and organized movement," which Dewey goes on to describe as "an artistic structure" [11, p. 38].

For Dewey, all well formed experiences are aesthetic. Aesthetic experiences are the golden mean between the opposing poles of aimless succession (plain experience) and mechanical connection (excessively rule-bound behavior), experienced subjectively as the humdrum and the rigidly constrained, respectively. In between is a purposive activity that meets some resistance before giving way to fulfillment or resolution. More specifically, Dewey characterizes aesthetic experiences as an interaction between doing and undergoing, that is, acting and being acted upon, which may include alternations of joy and pain, suffering and pleasure, before achieving their final unity, a 'felt harmony' in an experiential quality brought about by the resolution - and end - of the experience. Aesthetic experiences are not qualitatively different from all completed experiences; aesthetic experiences only differ in the degree of their "clarified and intensified development of traits that belong to every normally completed experience" [11, p. 46].

In anthropology, particularly the work of Victor Turner, Dewey's theories of aesthetic experience have been used to explore social conflict, using a theatrical metaphor. Turner describes a common pattern of social conflict, which begins with a breach in a previously harmonious community; a crisis that results from the breach; attempts to redress the breach and end the crisis; and ultimately a resolution in the form of reintegration or schism. Drama, he observes, tends to focus especially on the redress stage, and within it occurs a liminal phase (here is alludes to the work of van Gennep [37]). This liminal phase is characterized by dreams and nightmares, monsters and visions, sacred symbols and mysterious instructions, and sexual and gender ambiguity, among others [35]. As we will show, the liminal phase is a vital component of the virtual BDSM aesthetic.

In this genealogy, we see the elaboration of a theory of everyday experience and its relationship to art (Dilthey), which becomes understood in the context of a struggle that is ultimately pleasurable because of the meaning one derives from it (Dewey), which is further refined into a ritualized, theatrical staging of liminal banishment and social reintegration (Turner and van Gennep). This background contextualizes our formulation of the aesthetic, offered above, as a meaningful experience of struggle followed by resolution, characterized by a sense of self-transcendence and the urge to express or share the experience via cultural artifacts.

\subsection{Aesthetic Experience in HCI}

The distance from Turner's and van Gennep's liminal dreamvisions to the cold world of computer interfaces might, at first blush, seem great. Yet the gap has already been closed, with writers such as Brenda Laurel [21] exploring relationships between HCI and theatre and a number of HCI researchers appropriating the work of Dewey (e.g., $[14,23,28,36])$ to explore what some call 'experience design' and what others call 'aesthetic interaction', which, regardless of the name, share certain characteristics.

Genealogically, they rely on ideas and strategies drawn from the humanities, rather than the sciences. They tend to focus as the unit of analysis not on interfaces or even interactions (understood as a library of possible behavior sequences), but rather the everyday subjective experience of technology. 
Instead of privileging control, efficiency, and productivity, they focus on fulfillment, pleasure, delight, play, surprise, vitality, and sensual quality. They assume experience goes beyond the rational and the cognitive and is embodied, emotional, and socially constructed.

Building, for example, on pragmatic philosophers such as Dewey, McCarthy and Wright [23] identify four 'threads' of experience: the sensual, the emotional, the compositional, and the spatio-temporal. These threads are offered as a framework, as opposed to a model ("They are not fundamental elements of experience," p.79). This framework is intended to heighten people's sensibilities and ability to talk about the experience of technology. Criticism has replaced modeling, prediction, and experimentation; nuanced sensibilities have replaced scientific knowledge. HCI has moved away from developing tools for people to solve problems and instead has become HCI design, a discipline that produces and interprets interactive media experiences. In the language of Dilthey, software interfaces are the cultural expressions our contemporary society uses to frame and interpret individual experiences intersubjectively.

The anthropological notion of the liminal has not been developed significantly in the HCI literature. However, it is not hard to imagine how it might be. In those moments where software use transforms us, where we attempt to apply old ways of thinking in new digital contexts, or where we uncritically attempt to replicate real-world metaphors and ideologies in digital interactions, we produce the dreams, monsters, and otherworldly hybrids that can only be transient; examples perhaps include Microsoft Bob, the e-book with turnable pages, and the business Web site as electronic brochure, so common in the 1990s.

\subsection{Sadomasochism as Aesthetic Experience}

A central assumption of our project is that fetish sex practices can be aesthetic experiences, an assumption which might on the surface be surprising. Yet, when examined in light of postSchopenhauer notions of aesthetics and pragmatic notions of experience, BDSM can easily be described as aesthetic for its participants.

In a 1982 interview, the post-structuralist French philosopher and sociologist Michel Foucault describes S\&M in terms surprisingly similar to the language of Dewey. First, Foucault argues that (modern homosexual) sex has become, paraphrased in Dewey's terms, a mechanical connection devoid of interest.

What you have, then, is a situation where all the energy and imagination, which in the heterosexual relationship were channeled into courtship, now become devoted to intensifying the act of sex itself.... It is because the sexual act has become so easy and available to homosexuals that it runs the risk of quickly becoming boring, so that every effort has to be made to innovate and create variations that will enhance the pleasure of the act. [15; p. 225]

To make sex interesting, Foucault continues, S\&M partners coconstruct a certain kind of experience:

[S\&M] sexual relations are elaborated and developed by and through mythical relations. $\mathrm{S} \& \mathrm{M}$ is not a relationship between he (or she) who suffers and he (or she) who inflicts suffering, but between the master and the one on whom he exercises mastery. What interests the practitioners of $S \& M$ is that the relationship is at the same time regulated and open.... This mixture of rules and openness has the effect of intensifying sexual relations by introducing a perpetual novelty, a perpetual tension and a perpetual uncertainty.... [15; p. 226]

The salient characteristic of $\mathrm{S} \& \mathrm{M}$ is that both partners, dominant and submissive, both do and undergo, both experience risk and fear, and both exist in an uncertain space between rules and openness. The elaboration of ritualized, mythical sexual roles and codes of behavior help clarify and intensify the sexual experience. BDSM's monstrous imagestorture, violence, pain - are a by-product of the liminal state into which the partners place themselves. Combining Dewey and Foucault, we might say that if sex is an experience, $S \& M$ is one way of aestheticizing sex. Along these lines, as explored further below, the S\&M 'scene' (as an S\&M sexual encounter is tellingly known by its practitioners) clearly borrows the cultural logic of the theatre, with character roles, stages, props, and scripts.

While we are obviously not arguing that HCI is sadomasochistic (though we suspect a lot of people would probably agree with that claim), we began this project curious as to BDSM's ubiquity and social acceptability in Second Life. More specifically, we wondered how software interactions mediated and transformed sexual experiences. Minimally, the movement from 'vanilla' (non-fetish) sex to BDSM seems accelerated in Second Life (and we know from the MUDs of the past that the phenomenon is not limited to Second Life), which suggests that software interfaces may contribute to that acceleration.

\section{METHODOLOGY}

This study combines virtual ethnography, including observations and interviews with informants, with a study of hundreds of BDSM-related artifacts, including interactive props, fashions, and texts.

We began the data collection process towards the end of 2005 , in which principles from the three-part framework (visual, literary, and interaction) are used as a systematic way to identify, collect, categorize, and analyze groups of artifacts in a consistent manner.

Our initial (and primary) focus was on BDSM-themed artifacts, which we relied on for a number of reasons. The artifacts are readily available and free or inexpensive in Second Life. Additionally, they represent one of the least intrusive ways to study a community that for obvious reasons is somewhat guarded around outsiders. Third, included in the category of artifacts is a substantial corpus of practitioner-written texts made publicly available through various BDSM-themed libraries in-world. These texts number in the hundreds and include frank practical guides and how-to manuals, opinion pieces, transcripts of community events and rituals, and creative writings (such as poems). Second Life texts and props were compared with real-life BDSM practitioner guides (e.g., [1, 22, $38,40])$ as well as BDSM-themed fiction (e.g., [25, 30, 31]).

Informed by our artifact research, and after negotiations and IRB review, we also gained access to two communities, where we practiced participant observation, beginning in early 2007 . We observed educational events, for example, on how to be a submissive and on how to give one's master/mistress a bath, as well as community rituals, including collaring and branding ceremonies. These activities took place in dedicated BDSMthemed spaces, including clubs, bath houses, castles, taverns, and jungles, among others. Because experiences (of any kind) are socially and culturally constructed, group activities organized by fetish sex community members are a natural locus for artifact collection, which helped us verify our inferences 
from the earlier stages of the study and positioned us to understand what we were witnessing.

Beyond the artifacts obtained through fetish sex-themed events and activities, we also collect artifacts by accessing Second Life's search menu. A search on a total of 10 keywords (i.e., 'sub'. 'submissive', 'slave', 'Dom', 'Master', 'Mistress', 'bondage', 'bdsm', 'gor', and 'gorean') in the 'Classifieds' and 'Places' tabs, yields a list of Second Life vendors and entrepreneurial designers dedicated to create the embodied sexual experiences.

In all, we collected or observed data from/in the following sources:

- Fetish-based fashion, accessories, and animated body parts sold in 3D commerce vendors.

- Stores specializing in interactive sex toys and furniture.

- Weddings, branding, collaring, and ritualized ceremonies.

- Fetish-themed activities (e.g., dance contests, slave hunts, wrestling, etc.).

- Training sessions centered on BDSM education (e.g., classes on dominance, submission, bathing rituals, combat, food/drink service, role-play dos and don'ts, etc.).

- Community libraries housing member-created archives.

- Images posted at public sites.

- Second Life erotica (e.g., issues of the virtual porn magazines Slustler and Player).

We categorize and analyze these artifacts as they contribute to the development of aesthetic experiences in the sections below.

\section{VISUAL AESTHETIC}

BDSM is not just a sexual practice, but also a visual culture. In particular, BDSM and fashion have a well established history, made available to large audiences through the work of photographers such as Robert Mapplethorpe and Helmut Newton. In this section we explore its visual aesthetics, starting with its real-life roots and looking at their transformation into the virtual.

\subsection{BDSM in Mainstream Culture}

The visual aesthetic associated with BDSM is visible, if distorted, in mainstream culture. Common BDSM icons include leather, body piercings, tattoos for dressing up the body, and women in submission. Accessories include whips, paddles, rope and the like. Mainstream examples include Versace's controversial 'Bondage Collection' and Michelle Pfeiffer's outfit for Catwoman in Batman Returns, both from 1992 (Figure 1). In both cases, BDSM is referred to in order to add elements of danger and power to existing fashion [34]. Yet these appropriations are quite disconnected from actual BDSM experiences, replacing instead culturally charged but ultimately vacuous signifiers for the original experience.

\subsection{From Mere Allusion to Integrated Cultural Expression}

The mere presence of leather straps or a tattoo is not sufficient to constitute an artifact that is an expression of the BDSM experience. Instead, it is the arrangement of these elements, that is, their participation in a larger visual-semiotic system, that distinguishes between a scrap of leather on the floor and an art object, or cultural expression in Dilthey's vocabulary, connected with this subculture.
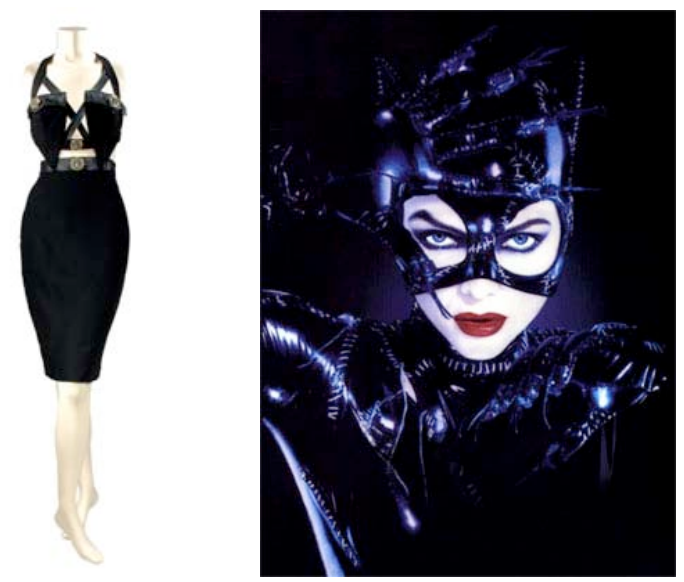

Figure 1. BDSM's visual aesthetic appears in mainstream culture, from Versace (left) to Hollywood (right).

The BDSM subculture has a number of aesthetic arrangements of its objects. For example, in the pony girl racing fetish (Figure 2 ), submissives are elaborately outfitted as ponies, forbidden to speak (visually symbolized by the bit), and displayed and raced as ponies in a competition [20]. They are posed in such a way as to accentuate their posteriors and when they move they prance in an equine manner. Japanese rope bondage is another BDSM art form, with its elaborate knots, rope patterns, and sculpted body positions. Indeed, fetish art is a popular topic of erotic photography books, such as $[10,17,33]$.

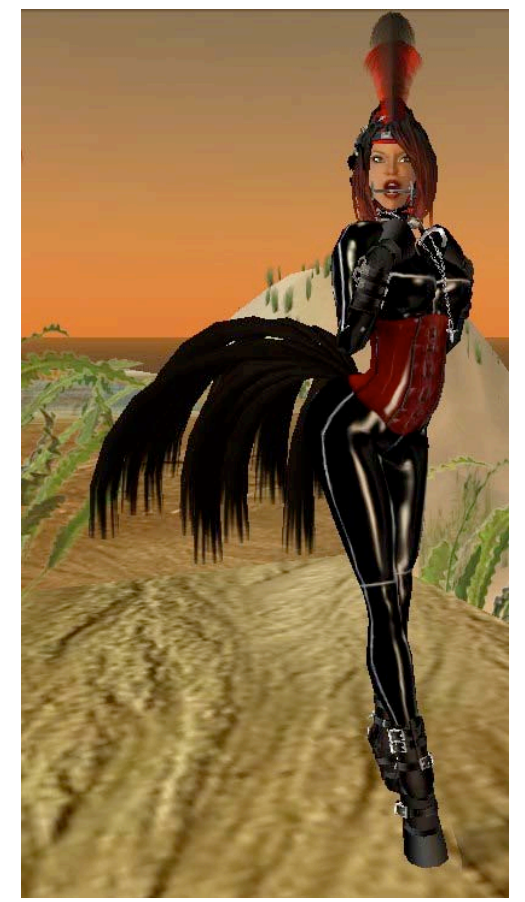

Figure 2. The pony girl fetish involves an arrangement of visual elements.

\subsection{BDSM Visuality's Mediation in Second Life}

All of these forms can be seen in Second Life; however, the realities of its authoring capabilities affect how and how often they appear. To construct visually rich interactions in Second Life, BDSM visual designers typically use 2D textures, created 
in Photoshop, to create the desired look. For example, whip marks are created as virtual tattoos on purchasable skins that avatars can put on when partners use whips during encounters. The use of floggers, a focus on buttocks and thighs, as well as carefully arranged stripes, bruises, and welts on the flesh/tattoos, all mutually-constitute the visual language of the spanking fetish, and it is fairly similar between the real-life and Second Life versions of the fetish. Pony racing attire and accessories are also readily available, in part because Second Life makes it easy to create clothing.

In contrast, Japanese rope bondage is harder to find in Second Life. We suspect one reason for this is that rope is difficult to model well. Presumably as a result, we have seen comparatively little evidence of this fetish in Second Life or sites about Second Life BDSM, even though it is popular in fetish photography.

The BDSM look is clearly a major visual resource for Second Life BDSM designers, and its influence appears not just in the clothes and props that they replicate from real-life BDSM. As Figure 3 shows, the automated vending machine from which avatars purchase the designs itself takes on the style of BDSM, as its heavy reliance on black and red extends the BDSM visual culture beyond articles of clothing and sex toys to Second Life e-commerce; this vendor communicates its participation in the subculture even before one really looks at any of the individual items for sale. Similarly, the relative inexpensiveness of virtual property means that users can create large, open-air BDSMthemed towns and villages, which extends the BDSM visuality out of the private spaces in which it appears in real life and comes to inform architecture and even zoning in large public spaces.

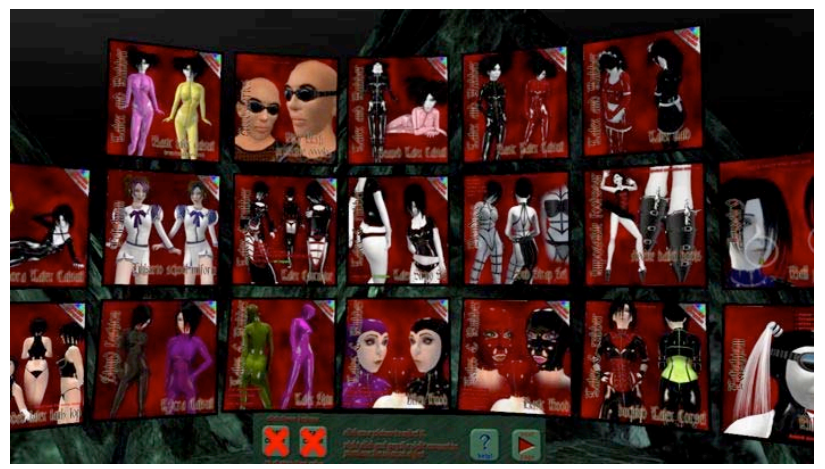

Figure 3. Second Life BDSM fashion has a coherent look, from the 'materials' to the color scheme.

One of the authors completed a study of Second Life's BDSM visual aesthetic in another article [3], which offered a visual analysis of over 400 randomly sampled user profile images from people who self-identify as members of a Second Life BDSM community. The study shows that the depiction of avatars in the self-portraits conveys much about both the individual personalities as well as a sense for the visual aesthetics of their communities, which are expressed through both social and photographic conventions. The analysis showed that while bodies belong to individuals, they are nonetheless defined and made meaningful by society. Physical characteristics (e.g., the skin tone, facial feature, makeup, etc.) are non-verbal signifiers that provide communication cues, adhering to the established BDSM notions of idealized gendered form, whether dominant or submissive.

The distinctive visual language of BDSM is by and large easily ported in-world and in some cases, such as the vendor and large
BDSM-themed physical spaces, extends beyond the boundaries to which it is typically limited in real life. The visual language is so recognizable and so immediate that it offers the quickestand most superficial-way for avatars to associate themselves with this lifestyle. At the same time, individual communities within the subculture have variations on the codes of beauty. For example, in some communities most of the women are fairhaired, while in others, a more goth look prevails; likewise some communities' appropriations of BDSM visuality are mediated by a medieval theme, while in others a cyberpunk theme is more evident. The visual aesthetic is by no means monolithic.

\section{LITERARY AESTHETIC}

One under-recognized aspect of BDSM, seen both in real life practice and in Second Life practice, is the literary organization of its events, which include both elaborate narratives and theatrical sexual encounters. Aspects of this literary aesthetic include the 'scene', the sophisticated role play, and the use of scripted rituals.

\subsection{The Scene}

The D/s scene is well documented in both academic [39] and practitioner $[38,40]$ literatures. In real life BDSM practice, the scene is a sexual event, negotiated and scripted in advance. It typically comprises a setting, roles, and a story. Negotiation includes a discussion of what each partner is willing and not willing to do (consent remains the key to all SM practice), the all-important safeword, as well as the shared construction of roles and a narrative. Weinberg [13] characterizes the scene as a 'keyed' transcription of another narrative, in which one story (e.g., the professor and the student) is systematically remediated into a BDSM scene.

Again, this characterization of the BDSM experience maps closely to the anthropological notion of experience, for example, as described in Turner [35] and echoed in [23], which sees meaning and value emerge relationally between the "preoccupying present experience" (in this case a sexual encounter), and past experiences, including experiences of cultural expressions (here, relationships of power and submission, such as one's own schooling or cultural depictions of schooling, from Victorian fiction to Pink Floyd's The Wall).

In Second Life both the scene and its pre-negotiation are common practice. However, the difference between avatar and one's physical self surely alters the nature of the limits to which people are willing to extend themselves. While safewords are common in Second Life BDSM practice, their significance as a physical safeguard is diminished, because the virtual submissive can always release her- or himself from any situation by detaching a scripted object, 'standing up' from a restrictive device, teleporting away, or even logging out.

\subsection{Role Play}

Role play is a critical part of the literary aesthetic of BDSM practice. In addition to the general role of dominant and submissive, partners often take up more specific, story-based roles consistent with the more general roles. Thus, a dominant may role play a professor, while the submissive role plays a student. Other pairings include master and slave, homeowner and maid/manservant, doctor and nurse, and so on.

The relationship between a dominant and a submissive is extensively discussed-and with quite a bit of consensus-in real life $[1,24,40]$ and Second Life (e.g., the hundreds of 'books' in the virtual libraries in BDSM hubs all over Second 
Life) practitioner discourses. Characterized in terms of balance-one writer [18] compares dominants and submissives to the black and white of the Chinese yin-yang symbol—both dominants and submissives have seriously articulated rights and responsibilities, limits as to what constitute appropriate behavior, attitudes, and expectations.

Observation of the roles results not only in a fulfilling relationship with the partner, but it makes it possible for submissives to enter a commonly, if vaguely, described special submissive state or condition, which we interpret to be a kind of self-transcendence or aesthetic rapture. Again, we reference Turner [p. 38]: "The moment of passage from disturbance into harmony is that of intensest life," and BDSM practitioners, enabled by Second Life technologies, are able to put themselves in extreme situations optimized to produce this passage from disturbance to harmony and create these intense and vital life experiences.

In addition to balance and transcendence, BDSM role play often enacts the aesthetic form of ornament. In addition to the visual ornament associated with BDSM accessories, submissives often play the role of ornaments for dominants, as sexually appealing adorers on the floor, on leashes, and even as parts of pieces of furniture, which places participants on the liminal threshold between the human and the inanimate (Figure 4). In Second Life's permissive culture, such displays are common and often accepted in public spaces, such as clubs; as a result, particlebased chain leashes, cages and cushions with submissive poses, and the like are easily available.

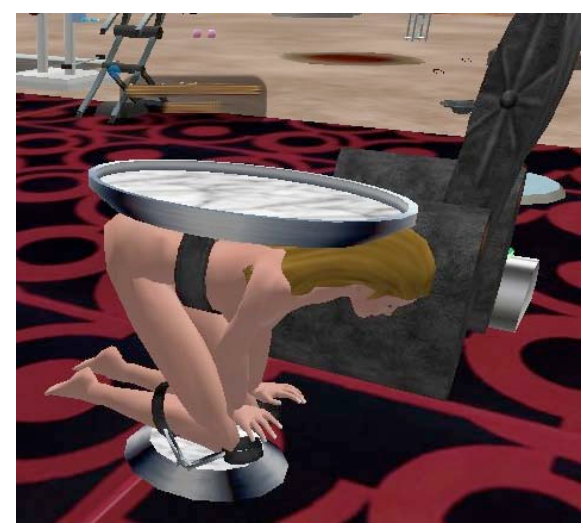

Figure 4. Second Life affords many ways to experience extreme liminality.

\subsection{Ritual}

Significant moments in BDSM relationships are often marked with a ritual, just like other human relationships. Common rituals include submissive training rituals, collaring and branding rituals, and rites of release (for the dissolution of relationships).

Collaring ceremonies (Figure 5) are particularly important, as they ritually cement the bond between two participants; they combine the cultural logic of weddings with the legality of property law, as the submissive more or less explicitly, becomes the property of the Dominant. In both real life and Second Life, collaring ceremonies often involving a script with a set of questions and the enactment of a contract.

The Second Life versions of this ritual have a few interesting twists. Some collaring rituals we have seen online inquire whether the submissive has 'alts' (other hitherto undisclosed avatar accounts) in Second Life or in other virtual worlds, such as ActiveWorlds. The intrusion of these questions in BDSM communities, such as Gor, which are based on a series of medieval fantasy novels [25], is strikingly non-diegetic; evidently, it is worth breaking the narrative illusion to ensure that the submissive's collaring is meaningful.

Another ritual takes a peculiar form in Second Life: the ritual of release. In the Gorean community, slaves who break off from their masters go through a rather extreme ritual: execution! In practice, this means that a representative of Gor confronts the slave, states in the chat window that he has killed her or him, and that she or he is now officially dead to Gor. This is followed up with banishment from the servers on which Gor resides, but not before a rather absurd dialogue in which the (dead) former slave expresses shock, demands to know the reasons for her or his execution, and often argues with the executioner about the appropriateness of the punishment.

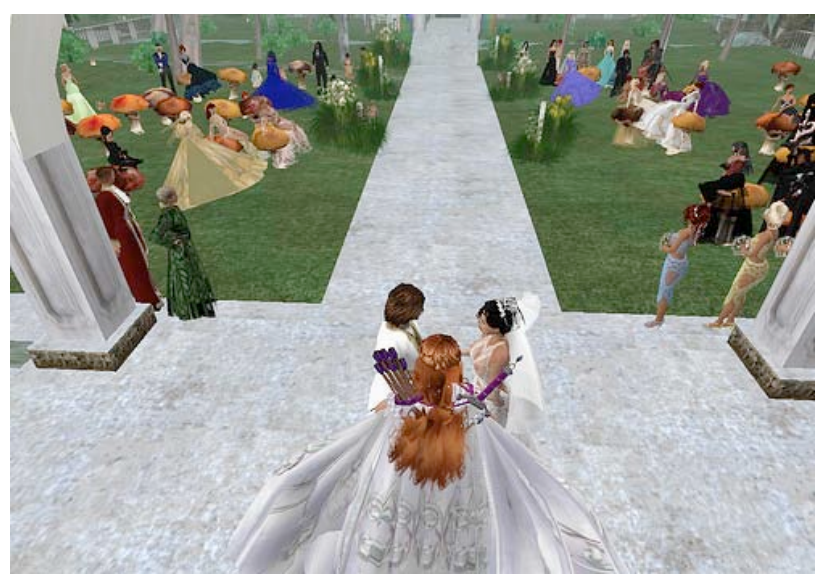

Figure 5. Collaring ceremonies are rituals that often resemble weddings.

The literary aesthetic of BDSM plays a complementary role to that of the visual aesthetic. The result is that through Second Life, participants can experience extreme sexual encounters enriched by nearly limitless narrative 'keys' that are made possible because just about any narrative can be visualized thanks to the inexpensive and easy availability of thousands of visually compelling fashions and props. Participants can push these visual performances to incredible extremes (e.g., the blood and gore fetish), because they and their avatars are in no physical danger. Of course, all this comes with the compromise that the thrill of real physical danger and pain is absent (though advances in teledildonics, or remotely controlled sex toys, may over time address that as well). Second Life BDSM, thanks to its software mediation, facilitates the aesthetic development and experiential intensification of sexual experiences, but it does so by foregrounding the visual and intellectual at the expense of the physical sense of touch, pain, and the adrenaline rushes that presumably accompany them. In short, the Second Life BDSM aesthetic experience is vicarious, but as we shall describe, vicarious in a special way.

\section{INTERACTION AESTHETIC}

Second Life BDSM is not all pictures and text. It is interactive, and that extends both to human-computer interaction and human-human interaction (which is mediated by computers). Second Life BDSM's human-computer interaction, therefore, and the unique experiences it affords, is a major part of its aesthetic. Understanding it should give us insights in how the community has designed and optimized aesthetic experiences 
using Second Life software, including the features of its client (chat, world viewer, inventory system, etc.), and the possibilities enabled by its authoring environment. The authoring environment contains tools and features to support easy $3 \mathrm{D}$ modeling, a native scripting language, and the ability to upload 2D bitmap textures and 3D poses and animations.

\subsection{From Sex Toys to Information Systems}

Understanding the role of interactive toys in Second Life BDSM scenes presupposes an understanding of object basics in this environment. Toys often bring together many if not all of the main components of the authoring tools: they are modeled out of simple 3D primitives, such as cubes and cylinders, which have imported 2D textures wrapped around them; scripts make them interactive, often providing clickable menus and outputting state information to the chat window; and they often place participants into poses.

Entire sexual interaction systems are created, such as the Xcite! series, which includes not only interactive genital attachments with menus and poses, but additional interactive objects and plug-ins (Figure 6), from collars to bull whips and cages, that all communicate with one another, such that receiving whips from the whip object may trigger an orgasm in the genital attachment, which in turn may produce particle semen and notification in the partners' respective chat windows.

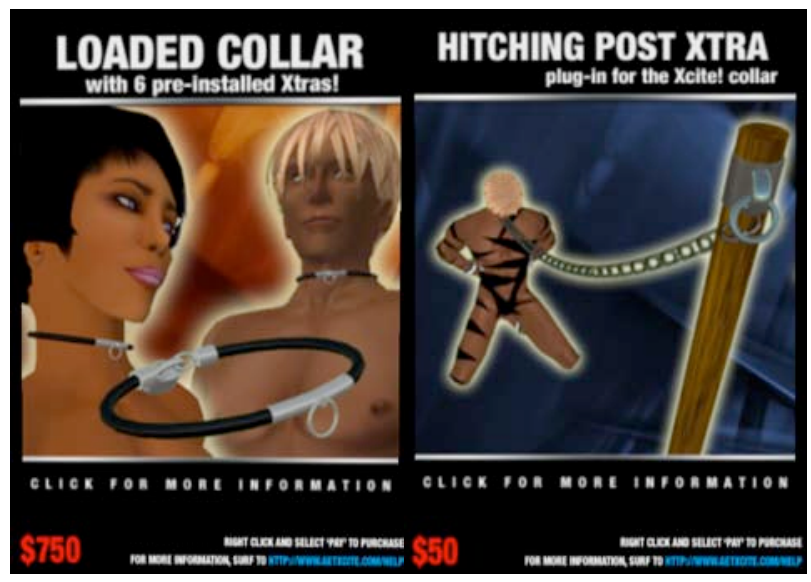

Figure 6. Individual toys become constituents of interoperable information systems.

In turn, these sexual information systems enable rather detailed simulation of certain fetishes. The pony girl fetish has already been mentioned, but another example is the kitty cat fetish, in which the submissive takes on the role of a kitten. In it, submissives have pointy cat ears and tails; their bodies are posed and animated such that they walk on all fours; they drink milk from bowls and eat virtual representations of canned cat food; and they use cat boxes. Scripts on the objects trigger appropriate animations, such as bending over to lick milk from the bowl or burying waste in the litter box. Of course, much descriptive activity also occurs via chat, including the expression of internal feelings, the giving of commands, and so on.

\subsection{Social Performance and Interactive \\ Spaces}

What is the subjective experience of these interactions? If Second Life BDSM were a purely vicarious experience, then the use of these toys would be akin to viewing fetish pornography. But by many accounts, it is different. Second Life users typically identify with their avatars; they don't just look at them. Several avatars have told us what we have also read on blogs: the first time one's avatar is caged or chained by another without explicit consent, there is a shock and a thrill. Even the authors of this paper, who are not BDSM practitioners in Second Life or real life, felt a shock and thrill when we were testing out various objects for the purposes of researching their functionality. Obviously, for many participants, this thrill never goes away, though over time its experience requires careful cultivation as repetition of the same interaction would have diminishing effects. The technologies enable the virtual self to enter liminal state-kitten, furniture, slave, pony girl-that enables two partners to explore the edges of human experience.

This leads us to the next issue, which is how participants cultivate these rushes by means of interactive objects, and in concert with the preexisting visual and literary aesthetics already described.

The answer lies in the construction of certain kinds of social performances that Second Life makes possible; in this, we are echoing the theorization of [16]. These performances are not simply interactions between the two (or more) participants in the encounter; they are developed, prescribed, and eventually taught through an institutionalized educational system in-world at the community level. Additionally, they depend on the spatio-temporal contexts in which they develop; thus, intimate encounters in dungeons naturally differ from intimate encounters in the bath. In short, communities, institutions, scripts for intimate interaction, and the settings in which they take place are mutually constituted-and every aspect of this dynamic is computer-mediated.

\subsection{The Baths of Gor: The Threads of an Experience}

One subset of BDSM practitioners follow the BDSM-themed cult fantasy novels of Gor [25], alluded to earlier, which take place in a medieval society in which many women are sexually enslaved to men. One of the communities that simulates these novels in Second Life recently held an education session (Figure 7) detailing the ritual of the Gorean bath, in which a slave, called a kajira in the novels, bathes and massages her or his Master or Mistress. This elaborate and highly codified interaction places two participants in a situation, both rulebound and open-ended, both intimate and communityembedded, both balanced and unequal in an interactive digital environment designed and optimized for aesthetic experience.

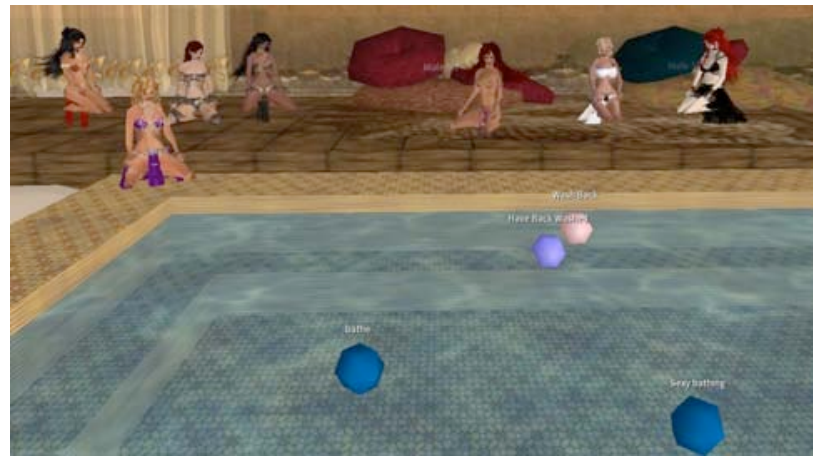

Figure 7. Kajirae (slaves) take a class on bathing their Masters. 
To unpack the complexity of this experience, after a brief summary of how it plays out, we borrow the four threads of experience from [23] as a means to understand and describe the experience.

In a Gorean bath interaction, a slave bathes and massages her or his Master/Mistress according to a certain established sequence, which, incidentally, is clearly modeled on ancient Roman bathing rituals. Note that the bathing ritual does not actually involve sex, though the experience is highly eroticized. The basic sequence is as follows: the Master/Mistress initiates the bath sequence. Once at the bath house, the slave generally takes control of the action. The partners enter a tub together, and the slave sponges the Master/Mistress, cleaning him or her. Next, the couple leaves the tub and goes to a massage table, where the slave massages the Master; this is made possible thanks to poses/animations built into the massage table that position the avatars' bodies correctly. After the massage, the slave scrapes the oil, dirt, and sweat from the Master/Mistress' body using a strigil (a metal tool used in the ancient world to scrape dirt and sweat from the body, modeled but not fully animated in Second Life). Finally, the couple returns to a second bath and the slave sponges off all the remaining oils and dirt.

\subsubsection{The Sensual Thread}

McCarthy and Wright, following Dewey, describe the sensual thread as that which connects people directly, palpably to the world. It enables the experienced mechanic to hear the tell-tale tick that indicates what's wrong with a car engine; it enables the child playing a GameBoy to become immersed in its world.

The sensual aspect of the Gorean bath experience in Second Life is so tied to Second Life's capabilities and limitations that it gives shape to the remaining threads. The most obvious limitation is that whereas Second Life excels with the visual, the bathing ritual is predominantly tactile and secondarily olfactory. This means that, as seen in Figure 7, that it is possible to recreate the visuals of a Gorean bath, but Second Life users playing Masters or Mistresses will never feel the gentle friction of the sponge as a slave works at a spot of dirt just under the collar bone; nor will the user playing a slave undergo the light soreness and fatigue in the biceps felt after giving a massage with oil and strigil; neither participant will smell the bath salts and soaps as they intermingle with steam, rising from the water to waft through humid, Mediterranean air toward the cracked stucco ceilings of the public bathhouse. To compensate for these fundamental sensual shortcomings, slaves must do as we just did: provide an evocative textual description instead.

The undercurrents of eroticism also follow. Slaves wear suggestive, but not entirely revealing, silks during the experience-and they don't take them off. Slaves are trained in Second Life to tell their Master or Mistress how their wet silks cling to their bodies, perhaps revealing the outline of a nipple or a hardening penis. Slaves are also trained to disrobe their Master or Mistress and to caress their thighs, and the Master or Mistress responds by emitting low growls. Yet none of this happens directly in the Second Life client. Second Life makes no distinction between wet and dry clothes. Avatars cannot actually growl. The eyes of the Masters and Mistresses during the bath are claimed to follow the slave's every move; yet Second Life does not capture this, either. Instead, in all these cases, the erotically charged elements occur through text chat as the participants co-construct an erotic play in real-time.

But Second Life is not a MUD, either. The avatars are visually realized, and they are sexually appealing. They are dressed in suggestive clothing. And perhaps above all they do appear together in intimate poses in the bath, with clouds of particle steam rising. The visuals are sufficient to set the stage, but it is the erotic writing, which elevates the experience from a poor man's simulation in participant-created Second Life to an intense sensual experience.

\subsubsection{The Emotional Thread}

The emotions are that quality that give an experience a value, coloring the experience and shaping its meanings, its value; emotions are not independent entities or states disconnected from experience. Clearly the participants in a bath scene cannot be reduced to rational minds processing streams of information provided by the senses. Rather, "the quality of felt life" for [23] is in "an emotional-volitional relationship with speaking and living others."

The emotional thread in the Gorean bath experience is above all intimate, an intimacy made possible by several characteristics. One is the vulnerability of disrobing together for a bath. Though the act of disrobing is not well visualized in Second Life (clothes just appear or disappear in binary fashion when put on or taken off), the closeness of the avatars bodies, when in a state of undress, in an intimate space such as a bathtub is conducive to feelings of intimacy, longing, and fulfillment.

Another is the practice of servitude, an obviously important BDSM general theme, which has been remediated away from public displays of human ownership and even humiliation (such as the serving trays Second Life submissives sometimes carry around with virtual drinks on them, some of which are designed to function even when the submissive's hands are bound) toward an exploration of servitude as an intimate, loving service. Likewise, the important BDSM theme of training is also given a gentle and intimate spin, rather than the more cruel forms that it so often takes.

\subsubsection{The Compositional Thread}

In the section on visual aesthetics, we argued that the BDSM aesthetic was more than just a collection of BDSM signifiers (tattoos, latex, etc.), but that it was an organized arrangement of these elements. When McCarthy and Wright characterize the compositional thread, they also seem to be focusing on how elements relate to one another.

In the words of the Gorean bathing instructor during the training session, "Bathing can be the most beautiful sight to behold as long as the teller is very descriptive in order to give a total visual of the actual bath." As we have alluded in the preceding discussion, the primary compositional medium of the Gorean bath in Second Life is the text narrative, produced by the storyteller-slave. This is due to limitations in the Second Life environment, which is capable of providing visuals to enrich a bath, but cannot do all the work on its own.

What kind of narrative is a Gorean bath? It is a narrative of increasing erotic tension, which is built up, yet controlled, over the course of the bath. Early erotic descriptions of the wet slave's body are tempered by the sponging and the soap smells, which are sensual but not explicitly sexual. Also, a series of planned disruptions also control the rising sexual tension: the first bath, the massage, and the second bath all must happen.

And above all, the sequencing and construction of the bath experience are very much in the control of the slave, while the Master or Mistress is primarily passive and reactive. This is a significant inversion of roles for standard BDSM behavior, because the slave's 'topping from the bottom' (which refers to 
situations in which slaves control conversations and dynamics) is not subversive, but is rather embedded in the experience.

The Gorean bath experience thus plays with the rules of BDSM relational dynamics, but it does so in formulaic ways, which at least guide the performance. Limitations of Second Life's technology also means that the mechanism through which the slave controls the interaction is not visual or animated, but is rather narrative and textual. Importantly, this fact does not prevent the bath experience from being a major part of Gorean BDSM play, which suggests that participants can have and in fact seek compelling experiences without the whole experience being animated with cutting edge graphics.

\subsubsection{The Spatio-Temporal Thread}

It is obvious that all social interaction occurs in space and time. But space and time are often understood as objective, abstract, unvarying categories, disconnected from human life, except inasmuch as human life takes place in them. McCarthy and Wright, following Bakhtin and in particular his theory of the chronotope [2], emphasize the extent to which space and time is subjectively experienced as well as the extent to which the categories of space and time are constitutive of what happens in them, rather than just containers holding those actions.

Here the significance of Second Life as an information system is most profound. By way of analogy, consider the player's capacity to make changes in World of Warcraft; they are practically nil. A given player may go on a quest and kill, as requested, an ogre named Ironfist, and they may bring his iron fist back as proof. Yet by the time the player has returned to the quest-giver, the ogre named Ironfist, along with his iron fist, have both reappeared in the same location they were always in, ready for the next player to come and complete the same quest (from the same quest-giver). Thus, while changes to the environment happen all the time, there is a sense in which the environment is radically static.

So it is in Second Life Gor. Players themselves may be highly mobile, taking on many sexual partners, coming and going at will, running away as slaves to become outlaw amazon-like huntresses, only to return as slaves, and run away again. The participants may take all sorts of actions, and these may offend other participants. But the simulation of Gor changes little: there is a caste system, in which there are Masters, Mistresses, and slaves, and there will never be a revolution that frees these slaves and sets up a representative government, which treats all citizens as equal, and enacts laws that ensures all labor is compensated. Cars, factories, and the printing press will not be invented. As an information system maintains state, so do many computerized simulations, and Gor is one of them.

The bath ritual takes place within this context. The slave taking the class learns how to perform the ritual. Through practice, she or he will presumably become better at it. But the relations between slave and Master/Mistress are largely proscribed and unchanging. The slave and Master/Mistress may break up, or a slave may serve someone else's Master/Mistress, but Masters will not start bathing themselves (avatars cannot become dirty anyway); there will not be a movement towards some notion of social justice; and no engineer is going to invent single-user showers or hand-sanitizer lotion.

\subsubsection{The Gorean Bath as Experience}

Technical limitations of Second Life, constraints embedded in BDSM roles, a visual aesthetic that integrates BDSM and Roman lifestyles combine to provide the basis of the bath scene. Yet these are insufficient to make the bath a good experience. The solution is to add a narrative dimension to it. Telling stories can be hard work, and it is passed onto the slaves, who are supposed to do all the work in a bath sequence anyway. But storytelling is a skill that presumably not all Gorean slaves have, and thus a narrative formula combined with explicit teaching fills in the gaps. Yet giving this sort of control to slaves is an unusual inversion of the normal order of Gorean Master-slave interactions, and so in a way Second Life's limitations give rise to a ritualized topping from the bottom interaction that once again plays at the boundaries of human relations, allowing its participants to aestheticize intimate interactions.

\section{THE VIRTUAL BDSM AESTHETIC}

Second Life BDSM, not unlike real-life BDSM, represents the convergence of several powerful socio-cultural forces to construct highly stylized human experiences. These experiences push at the boundaries of the socially acceptable, and in so doing position their participants in extreme situations where social mores, while still relevant, no longer clearly apply, and where the position of liminality is constructed and adopted to enable practitioners to act and undergo extreme experiences, which, when fulfilled, lead to intense pleasures.

Second Life, by providing its own world, rules, technologies, and interfaces, mediates the practices of BDSM within it. In that mediation, Second Life helps fashion its practices. Second Life BDSM is unquestionably practiced as a hybrid between a fully realized and animated video game and an old-fashioned text-based MUD. That is, Second Life BDSM is highly visual and yet also highly textual. This is not a rational decision on the part of its practitioners; it is conditioned response to the environment it is in.

This phenomenon should be of interest to HCI designers and practitioners, because the Second Life BDSM community has optimized itself and its technologies for the kinds of highly aestheticized experiences it values. No single designer designed them. They evolved over time in the hands of thousands of user-designers who are passionate about having these kinds of experiences. Whatever we think of BDSM-in real life or online-its experiences are important, vital, meaningful, and above all aesthetic for its participants. That they have figured out how to design such powerful experiences, including how they figured it out and above all what they designed, can teach us much about experience design.

In the case of Second Life BDSM, that mixture was to bring together Second Life's visual strengths with the powerful visual language BDSM has been developing for decades throughout culture, from high fashion to gay leather movements. Where Second Life is weak-animation and capturing subtle changes in state (such as wet or dry) - the community developed and disseminated narrative formulas. In the meantime, increasingly sophisticated and interoperable toys such as collars and paddles add visual richness and occasionally improve support for simulated fetishes, such as pony girl races. But though these improve certain elements, they are not transformative; the practice of BDSM has changed little in Second Life in the two years we have been researching it.

Real-life BDSM often involves physical pain and danger; as with all forms of real-life sex, it is obviously embodied. Second Life's sex, thanks to the ambiguous relationships between avatars and the human players behind them, is more stylized, and often it operates in ways that would be deadly in the real world. It is more mediated, constantly invoking fashion, theatre, storytelling, BDSM literature, and so in a way it must be less 
physical and more cerebral. But as [1] says, "the brain is the largest human sex organ [so] BDSM is truly a mind-game." It may be that Second Life offers a new interface to a classic, taboo aesthetic; but as it does so, its BDSM community also transforms that aesthetic in subtle, yet profound ways, providing compelling experiences through software to an international population of sexual beings, craving intense experience, driven by desire, guided by formulas, and protected by anonymity.

\section{REFERENCES}

[1] Abernathy, C. Miss Abernathy's Concise Slave Training Manual. Greenery Press, San Francisco, 1996.

[2] Bakhtin, M. The Dialogic Imagination: Four Essays. [M. Holquist (ed.); C. Emerson and M. Holquist (trans.)]. University of Texas Press, Austin, TX, 1981.

[3] Bardzell, S. The submissive speaks: The semiotics of visuality in virtual BDSM fantasy play. In Proceedings of the 2006 ACM SIGGRAPH symposium on Videogames. ACM Press, New York, NY, 2006, 99-102.

[4] Bell, G., Brooke, T., Churchill, E., and Paulos, E. Intimate ubiquitous computing. Proceedings of Ubicomp Workshop. 2003, 3-6.

[5] Blythe, M., and Jones M. Human computer (sexual) interactions. Interactions, 11, 5 (2004), 43-46.

[6] Blythe, M., Wright, J., McCarthy, J., and Bertelsen O. Theory and method for experience-centered design. In $\mathrm{CHI}$ 2006: Extended abstracts of the SIGCHI conference on Human factors in computing systems. ACM Press, New York, NY, 2006, 1691-1694.

[7] Brewer, J. Kaye, J., Williams, A., and Wyche, S. Sexual interactions: Why we should talk about sex in HCI. In $\mathrm{CHI}$ 2006: Extended abstracts of the SIGCHI conference on Human factors in computing systems. ACM Press, New York, NY, 2006, 1695-1698.

[8] Bruner, E. Experience and its expressions. In V. Turner, and E. Bruner (eds.), The anthropology of experience. University of Illinois Press, Urbana, IL, 1986.

[9] Buchenau, M., and Suri, J. Experience prototyping. In Proceedings of the conference on Designing interactive systems: processes, practices, methods, and techniques (DIS 2000). ACM Press, New York, NY, 2000, 424-433.

[10] Delves-Broughton, E. Kinky Couture. Goliath, Frankfurt, Germany, 2003.

[11] Dewey, J. Art as Experience. A Perigee Book, New York, 1934.

[12] Dickie, G. Introduction to Aesthetics: An Analytic Approach. Oxford UP, New York, 1997.

[13] Dourish, P. Where the Action Is: The Foundations of Embodied Interaction. MIT Press, Cambridge, MA, 2001.

[14] Fishwick, P., Diehl, S., Prophet, J., and Löwgren J. Perspectives on aesthetics computing. Leonardo, 38, 2 (2005), 133-141.

[15] Foucault, M. Sexual choice, sexual act. James O'Higgins, interviewer and trans. (1982). In Lotringer, S (ed.), Foucault live. Semiotext(e), New York, 1989, 211-231.

[16] Goffman, E. The Presentation of Self in Everyday Life. Penguin, Harmondsworth, 1969.

[17] Higgs, L. Fetish. Goliath, Frankfurt, Germany, 2000.
[18] Jade. Yin and Yang, 1996. http://www.castlerealm.com/library/

[19] Jade. A Submissive Owner's Manual. 1998. $\mathrm{http}: / / \mathrm{www}$. castlerealm.com/library/

[20] Julianna (n.d.). Pony sub Training. http://www.bankhead.net/BlackRoseCastle/

[21] Laurel, B. Computers as Theatre. Addison-Wesley, Reading, MA, 1991.

[22] Lorelei. The Mistress Manual. Greenery Press, San Francisco, 2000.

[23] McCarthy, J., and Wright, P. Technology as Experience. The MIT Press, Cambridge, Massachusetts, 2004.

[24] Moser, C., and Madeson, J. Bound to be Free. Continuum, New York, 2005

[25] Norman, J. Tarnsman of Gor. Wildside Press, Holicong, PA, 1967.

[26] Norman, D. Emotional Design: Why We Love (or Hate) Everyday Things. Basic Books, New York, NY, 2004.

[27] Paviova, I. Porncraft: WoW Erotica and Roleplay Forums. 2005. http://www.mmorgy.com/2005/11/

[28] Petersen, M., Iversen, O., Krogh, P., and Ludvigsen, M. Aesthetic interaction: A pragmatist's aesthetics of interactive systems. Proceedings of the conference on Designing interactive systems: processes, practices, methods, and techniques (DIS 2004). ACM Press, New York, NY, 2004, 269-276.

[29] Picard, R. Affective Computing. MIT Press, Cambridge, MA, 1997.

[30] Réage, P. The Story of O. Ballantine Books, New York, 1973.

[31] Rice, A. The Sleeping Beauty Novels. A Plume Book, New York, 1990.

[32] Ruberg, B. Cyberporn Sells in Virtual World. 2005. http://www.wired.com/news/culture/0,69878$0 . h t m l ? t w=w n \_t o p h e a d \_2$

[33] Speliotis, S. Asia Bondage. Goliath, Frankfurt, Germany, 2003.

[34] Steele, V. Fifty Years of Fashion: New Look to Now. Yale University Press, New Haven, 1997.

[35] Tuner, V. Dewey, Dilthey, and Drama: An essay in the anthropology of experience. In V. Turner, and E. Bruner (eds.), The anthropology of experience. University of Illinois Press, Urbana, IL, 1986.

[36] Udsen, L., and Jørgensen. The aesthetic turn: Unraveling recent aesthetic approaches to human-computer interaction. Digital Creativity, 16, 4 (2005), 205-216.

[37] Van Gennep, A. The Rites of Passage. University of Chicago Press, Chicago, 1960.

[38] Warren, J. Safe, Sane, Consensual, and Fun. Greenery Press, San Francisco, 2002.

[39] Weinberg, T. Sadism and masochism. In T. Weinberg (ed.), Studies in dominance \& submission. Prometheus Books, Amherst, New York, 1995, 119-137.

[40] Wiseman, J. SM 101. Greenery Press, San Francisco, 1996. 
